\title{
Management of carbapenem-resistant Acineto- bacter baumannii epidemic in an intensive care unit using multifaceted intervention strategy
}

\author{
Jeongmin Hong ${ }^{1}$, Ok Jeom Jang ${ }^{2}$, Mi Hui Bak², Eun Hwa Baek², Ki-Ho Park ${ }^{3}$, Sun In Hong 4 , \\ Oh-Hyun $\mathrm{Cho}^{1,2}$, and In-Gyu Bae $\mathrm{B}^{1,2,4}$
}

\begin{abstract}
${ }^{1}$ Department of Internal Medicine, ${ }^{2}$ Infection Control Office, Gyeongsang National University Hospital, Jinju; ${ }^{3}$ Department of Internal Medicine, Kyung Hee University Hospital, Seoul; ${ }^{4}$ Department of Internal Medicine, Gyeongsang National University Changwon Hospital, Changwon, Korea
\end{abstract}

Received: October 8, 2016

Revised : January 14, 2017

Accepted: January 15, 2017

\section{Correspondence to}

Oh-Hyun Cho, M.D.

Department of Internal

Medicine, Gyeongsang National

University School of Medicine, 79

Gangnam-ro, Jinju 52727, Korea

Tel: $+82-55-750-8745$

Fax: +82-55-755-9078

E-mail: zenmd@naver.com

This work was presented in part at ID week, San Diego, 7 to 11

October 2015 (abstract number: 52872).
Background/Aims: We evaluated the effects of a multifaceted intervention for controlling a carbapenem-resistant Acinetobacter baumannii (CRAB) epidemic in an intensive care unit (ICU) with no restrictions on carbapenem use.

Methods: This study was conducted in a medical ICU between April 2012 and June 2016. The baseline infection control programs included surveillance cultures, contact precautions, and environmental cleaning. However, increases in the detection of CRAB isolates beginning in May 2013 led to the implementation of a new protocol, consisting of universal glove and gown use with daily chlorhexidine bathing for all patients in combination with baseline programs. The efficacy of the intervention was subjected to interrupted time series (ITS) analysis.

Results: The multifaceted intervention led to a decrease in the incidence of CRAB from 17.65 to 0.89 cases per 1,000 patient-days, during the study period $(p<0.001)$. ITS analysis revealed a significant change in the trend of CRAB incidence $(-0.413$, $p<0.001)$. Over this same period, mean monthly use of carbapenems increased from $143.54 \pm 95.73$ to $204.95 \pm 78.43$ doses per 1,000 patient-days $(p=0.018)$.

Conclusions: Our multifaceted intervention reduced the incidence of CRAB acquisition in an ICU where carbapenem use was not restricted.

Keywords: Acinetobacter baumannii; Carbapenem resistance; Chlorhexidine; Intensive care units

\section{INTRODUCTION}

Carbapenem-resistant Acinetobacter baumannii (CRAB) is a major nosocomial pathogen in intensive care units (ICUs), and infections caused by CRAB are considered difficult to treat and control [1]. Infection-control measures including hand hygiene, active surveillance cultures (ASCs), contact precautions (CPs), and environ- mental cleaning are generally recommended to reduce the transmission of CRAB in ICUs [2-4].

Following a spike in the isolation of CRAB within our ICU in May 2013, our infection-control team initially sought to control the epidemic by intensifying existing infection-control strategies, but the incidence of CRAB continued to increase. Therefore, advanced infectioncontrol measures, including universal glove and gown 
use for all patient contact along with chlorhexidine bathing were introduced in October 2013. While these procedures have been reported in the context of methicillin-resistant Staphylococcus aureus (MRSA) and vancomycin-resistant enterococci, few studies have focused on CRAB [5-7]. Furthermore, although carbapenem restriction has been identified as an important measure for controlling CRAB transmission, physicians are often reluctant to shorten the use of these antibiotics, particularly for critically ill patients commonly seen in ICU settings $[4,8,9]$. Here, we describe our experience implementing these advanced infection-control measures in a medical ICU where carbapenem use was not restricted.

\section{METHODS}

\section{Study design and hospital setting}

The study was conducted in a 13-bed medical ICU at Gyeongsang National University Hospital, an 890bed teaching hospital in Jinju, Republic of Korea. The ICU has six single rooms and one open bay consisting of seven beds spaced approximately $2 \mathrm{~m}$ apart. The nurse-to-patient ratio was 1:3, with one environmental management assistant assigned to the ICU. Two infectious-disease specialists and three infection-control nurses supervised and assisted infection control in the ICU. All patients admitted to the ICU between April 2012 and June 2016 were included in this study. To assess the impact of advanced intervention programs, we retrospectively analyzed the rate of CRAB infection or colonization between two periods, a baseline 18-month period (April 2012 to September 2013; period 1) prior to implementation of advanced infection control procedures, and a 32-month intervention period (November 2013 to June 2016; period 2). The 1-month (October 2013) introductory period during which these new interventions were implemented was not included in the analysis. No antibiotic-restriction policies were used during the course of this study.

This retrospective study included the institution's healthcare infection data, which were regularly collected and reported by the hospital's infection control committee as a part of the institution's quality-improvement programs. Given the nature of the data collection, Institutional Review Board approval was not needed.

\section{Infection control programs}

During period 1, ASC for CRAB was performed for all patients within 24 hours of admission to the ICU using nasal swabs. Before June 2012, follow-up ASC was performed once per week only in patients with positive CRAB cultures; after June 2012, follow-up ASC was performed once per week regardless of CRAB isolation throughout period 1, to match the interval of ASC used for MRSA detection. As no cases of CRAB were detected by ASC between April 2012 and June 2012, we included this period as part of study period 1 .

Clinical culture for CRAB was performed when infections were clinically suspected. CRAB-positive patients were moved to single rooms or a cohort area with other CRAB patients, and managed via $\mathrm{CP}$, until three consecutive follow-up cultures were negative for CRAB. CP included placement of a warning post at the bedside, strict adherence to hand hygiene protocols, the use of vinyl gowns and gloves, and dedicated medical equipment for patient care of index cases. Hand hygiene using alcohol gel was promoted, and environmental cleaning of the cohort areas was performed once per day using sodium hypochlorite solution. Feedback on compliance with hand hygiene and $\mathrm{CP}$, and data on CRAB infection or colonization in the ICU, were given to the nursing staff and ICU physicians by an infection-control team biweekly through education rounds.

During period 2, the following advanced infectioncontrol measures were added to the previous programs in period 1. Beginning in October 2013, ASC was performed from both the anterior nares and throat using a sterile swab; ASC from both the inguinal and axilla areas using an additional swab was added beginning in August 2014. Follow-up ASC was performed weekly and at discharge throughout period 2. Universal glove and gown use during all patient contact, combined with chlorhexidine bathing regardless of ASC results, were also added based on a previous study suggesting insufficient sensitivity of ASC for CRAB [10]. These measures included wearing a disposable polyvinyl apron and gloves upon contact with any patient in the ICU. Moreover, all ICU patients were bathed with $2 \%$ chlorhexidine gluconate (CHG) solution daily on weekdays. If CRAB was identified from any cultures, CHG bathing was continued through the weekend until three consecutive followup cultures were negative. The bathing was conducted 
with washcloths soaked in CHG solution except above the neck, on the perineum, or on open wounds. Because this enhanced program was performed regardless of CRAB isolation, we considered the effects of changing ASC methods on the control of CRAB less significant. Therefore, when analyzing advanced interventions, we did not discriminate the period during which the ASC methodology was changed from that of period 2 .

\section{Definitions and outcomes}

All adults admitted to the ICU with CRAB identified by ASC and/or clinical cultures were included in our results. Multiple positive samples from the same patient were considered a single episode. Admission-prevalent cases were defined as an ASC or clinical culture positive for CRAB from a sample $<48$ hours after ICU admission, or an ASC or clinical culture positive for CRAB during the same hospitalization before admission to the ICU [11]. Incident cases were defined as CRAB colonization or infection identified from clinical cultures $>48$ hours after ICU admission and initial surveillance, or clinical cultures negative for CRAB at ICU admission. The admission-prevalence density of CRAB infection or colonization was defined as the number of prevalent cases per 1,000 patient-days (PD) in a given month. The incidence density of CRAB infection or colonization was defined as the number of incident cases per 1,000 PD in a given month. The change in the incidence density of CRAB detection based on clinical cultures between these two periods was the primary outcome of this study. Secondary outcomes included the change in monthly incidence density of CRAB infection or colonization from ASC and/or clinical cultures, and the monthly incidence density of MRSA infection or colonization from clinical culture during the study period. Because changes in ASC methods might have affected the observed incidence between the two study periods, we considered the incidence based on clinical cultures to be more reliable for identifying any effects associated with the advanced interventions in period 2.

The consumption of antipseudomonal carbapenem (doripenem, imipenem, and meropenem) was calculated as the defined daily dose (DDD) per 1,000 PD to evaluate whether any significant changes occurred during the study period [12].

\section{Microbiological methods}

We identified A. baumannii and tested antibiotic susceptibility using a Vitek-2 system (bioMérieux, Durham, NC, USA). Susceptibility results were interpreted according to the Clinical and Laboratory Standards Institute guidelines [13]. CRAB isolates were defined as A. baumannii isolates with a minimum inhibitory concentration $>4 \mu \mathrm{g} / \mathrm{mL}$ for imipenem or meropenem.

\section{Statistical analysis}

To investigate the effects of the intervention, we used an interrupted time series (ITS) analysis using a segmented Poisson regression. This method is helpful for detecting changes in the level (i.e., intercepts: abrupt intervention effect) and trend (i.e., slopes: gradual intervention effect) of clinical incidence after the launch of advanced interventions [14]. A segmented Poisson regression model is represented by the equation: $\ln (\lambda)=\beta_{\mathrm{o}}+\beta_{1}(\mathrm{~T})+\beta_{2}(\mathrm{I})$ $+\beta_{3}\left(T^{*}\right)$, where $T$ is the month, $I$ is intervention, $T^{*}$ is months after intervention, $\beta_{1}$ is the trend prior to intervention, $\beta_{2}$ is the change in level, and $\beta_{3}$ is the difference between the preintervention and postintervention trends $[14,15]$. First-order autocorrelation was tested using the Durbin-Watson statistic test. All tests were twotailed, with $p<0.05$ considered statistically significant. Statistical analyses were performed with $\mathrm{R}$ version 3.2.3 (R Foundation for Statistical Computing, Vienna, Austria).

\section{RESULTS}

A total of 2,149 patients were admitted to the medical ICU (729 patients in period 1 and 1,420 in period 2) over the course of the study period. No significant differences in utilization ratio of central venous catheters and ventilators were found between the two periods; however, the median length of ICU stay was longer in period 1 than in period $2(p<0.001)$. Of the 2,149 patients in our cohort, $246(11.4 \%)$ exhibited some form of CRAB colonization or infection during their time in the ICU (44 patients in period 1 and 202 patients in period 2). During period 1,18 of the 44 CRAB-positive patients (40.9\%) were identified by surveillance cultures (three by ASC alone, 15 by both ASC and clinical cultures). The remaining 26 CRAB-positive patients were identified by clinical cul- 
Table 1. Demographic and microbiological characteristics during the study period

\begin{tabular}{|c|c|c|c|}
\hline Variable & Period $1(n=729)$ & Period $2(n=1,420)$ & $p$ value \\
\hline Age, yr & $71(59-78)$ & $70(57-78)$ & 0.208 \\
\hline Male sex & $451(61.9)$ & $1,006(64.2)$ & 0.285 \\
\hline Patients no. with stays $>48 \mathrm{hr}$ & $583(80.0)$ & $1,140(72.8)$ & $<0.001$ \\
\hline Ventilator utilization ratio ${ }^{\mathrm{a}}$ & $0.76(0.74-0.82)$ & $0.75(0.72-0.78)$ & 0.462 \\
\hline Central line utilization ratio ${ }^{\mathrm{a}}$ & $0.65(0.62-0.71)$ & $0.63(0.59-0.68)$ & 0.265 \\
\hline Length of ICU stay, day & $5(3-10)$ & $4(1-9)$ & $<0.001$ \\
\hline Patient-days, total & 6,418 & 11,545 & NA \\
\hline No. of patients yielding CRAB isolates & $44(6.0)$ & $202(14.2)$ & $<0.001$ \\
\hline \multicolumn{4}{|l|}{ Sites of CRAB isolation ${ }^{\mathrm{b}}$} \\
\hline Surveillance cultures & $18(2.5)$ & $160(11.3)$ & $<0.001$ \\
\hline Respiratory tract & $38(5.2)$ & $143(10.1)$ & $<0.001$ \\
\hline Blood & $6(0.8)$ & $19(1.3)$ & 0.400 \\
\hline Wound & $3(0.4)$ & $12(0.8)$ & 0.416 \\
\hline Other ${ }^{c}$ & $5(0.7)$ & $5(0.4)$ & 0.321 \\
\hline No. of admission prevalent cases of CRAB ${ }^{\mathrm{d}}$ & $18(2.5)$ & $83(5.8)$ & $<0.001$ \\
\hline No. of incident cases of $\mathrm{CRAB}^{\mathrm{e}}$ & $26(3.6)$ & $88(6.2)$ & 0.011 \\
\hline $\begin{array}{l}\text { No. of incident cases of CRAB including both surveillance } \\
\text { and clinical cultures }\end{array}$ & $26(3.6)$ & $119(8.3)$ & $<0.001$ \\
\hline \multicolumn{4}{|c|}{$\begin{array}{l}\text { Values are presented as median (interquartile range) or number (\%). } \\
\text { ICU, intensive care unit; NA, not applicable; CRAB, carbapenem-resistant Acinetobacter baumannii. } \\
\text { a Device utilization ratio is calculated by dividing the number of device-days by the number of patient-days. } \\
\text { b Multiple positive samples from the same site were considered as a single isolation. } \\
{ }^{c} \text { Includes cultures from urine, ascites, pleural effusions, central venous catheter tip. } \\
\text { d Cases identified from surveillance or clinical cultures taken less than } 48 \text { hours after ICU admission, or positive CRAB during } \\
\text { the same hospitalization before admission to the ICU. } \\
\text { e }\end{array}$} \\
\hline
\end{tabular}

tures alone. During period 2, 160 of 202 CRAB-positive patients (79.2\%) were identified by surveillance cultures (49 by ASC alone, 111 by both ASC and clinical cultures) (Table 1).

The results of segmented Poisson regression analysis are shown in Table 2 and Fig. 1 . During period 1,18 patients were identified as CRAB positive on admission to the ICU, resulting in an admission-prevalence density of 2.81 cases per 1,000 PD. Meanwhile, 83 patients were identified as CRAB positive on admission to the ICU, for an admission-prevalence density of 7.19 cases per 1,000 PD during period 2. ITS analysis indicated a significant trend change in admission-prevalence density of CRAB infection or colonization between the two periods (-0.185; 95\% confidence interval [CI], -0.310 to $-0.059)$.

Only three cases of ICU-acquired CRAB were iden- tified between April 2012 and March 2013, followed by a spike in CRAB acquisition to 17.65 cases per 1,000 PD in the last quarter (from June 2013 to September 2013) prior to implementation of advanced interventions. Meanwhile, the incidence density of CRAB acquisition decreased from 11.85 cases per 1,000 PD in the first quarter after implementation of advanced interventions to 0.89 cases per 1,000 PD in the last quarter (from April 2016 to June 2016) of period 2, although some upswings in incidence were observed during this period. ITS analysis showed that the introduction of advanced infection-control measures was associated with a significant change in the trend of CRAB cases per 1,000 PD (-0.413; 95\% CI, -0.585 to -0.240). These findings indicate that the significant increasing slope of CRAB incidence (0.347; 95\% CI, 0.175 to 0.517 ) in period 1 was changed to a decreasing slope of -0.066 (95\% CI, -0.309 to 0.177) CRAB 


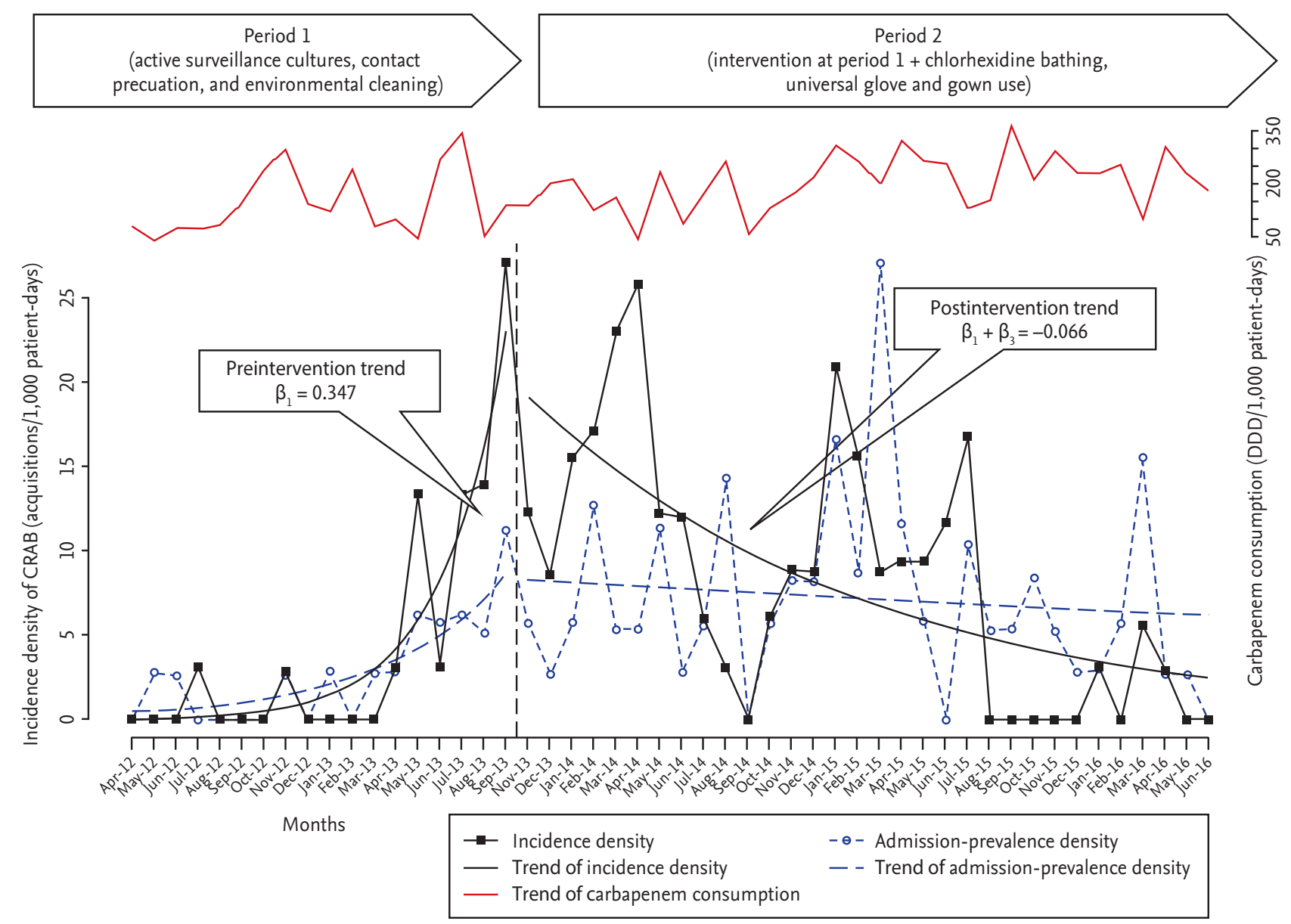

Figure 1. Changes in the incidence and admission-prevalence density of carbapenem-resistant Acinetobacter baumannii (CRAB) infection or colonization, and carbapenem consumption during the study preiod. DDD, defined daily dose.

cases per 1,000 PD after implementation of advanced control measures. The incidence density of CRAB infection or colonization including both clinical cultures and ASC showed a similar change after intervention. The incidence density of MRSA infection or colonization also showed a change in the level $(-0.137 ; 95 \% \mathrm{CI},-1.023$ to 0.750 ) and trend (-0.067; 95\% CI, -0.150 to 0.011 ) of disease incidence after intervention, although these differences were not significant (Table 2).

Hand hygiene adherence significantly increased from $72.3 \%$ in period 1 to $83.3 \%$ in period $2(p<0.001)$, but compliance with contact precautions was not significantly different between the two periods $(63.7 \%$ in period 1 vs. 68.5\% in period 2, $p=0.399)$. Meanwhile, monthly mean carbapenem use increased significantly from $143.54 \pm 95.73$ DDD per 1,000 PD in period 1 to $204.95 \pm$ 78.43 DDD per 1,000 PD in period $2(p=0.018)$.

\section{DISCUSSION}

Two important features underlying A. baumannii pathogenesis are its ability to survive for prolonged periods on dry surfaces and its propensity for resistance to multiple antimicrobials, resulting in numerous outbreaks or it being endemic $[3,16]$. Several major intervention programs in epidemic and endemic settings have identified methods capable of limiting the spread of CRAB, including cohort isolation, improved hand hygiene, and environmental disinfection $[4,16,17]$. However, in instances where CRAB isolation continues to increase despite these basic interventions, infection-control teams must consider additional intervention strategies, many of which remain inconclusive. Here, we showed that a multifaceted infection-control measure including universal glove and gown use during all patient contact 
Table 2. Results of segmented Poisson regression analysis of an interrupted time series exploring the effect of multiple interventions

\begin{tabular}{|c|c|c|c|c|c|c|}
\hline \multirow{2}{*}{ Variable } & \multicolumn{2}{|c|}{ Trend before INT } & \multicolumn{2}{|c|}{ Change in level after INT ${ }^{\mathrm{a}}$} & \multicolumn{2}{|c|}{ Change in trend after INT ${ }^{\mathrm{b}}$} \\
\hline & $\beta_{1}(95 \% \mathrm{CI})$ & $p$ value & $\beta_{2}(95 \% \mathrm{CI})$ & $p$ value & $\beta_{3}(95 \% \mathrm{CI})$ & $p$ value \\
\hline $\begin{array}{l}\text { Admission-prevalence } \\
\text { density of CRAB }\end{array}$ & $0.175(0.052$ to 0.298$)$ & 0.005 & $0.164(-0.673$ to 1.001$)$ & 0.700 & $-0.185(-0.310$ to -0.059$)$ & 0.004 \\
\hline Incidence density of CRAB ${ }^{\mathrm{d}}$ & $0.347(0.175$ to 0.517$)$ & $<0.001$ & 0.289 (-0.409 to 0.987$)$ & 0.417 & $-0.413(-0.585$ to -0.240$)$ & $<0.001$ \\
\hline $\begin{array}{l}\text { Incidence density of CRAB } \\
\text { including both ASC and } \\
\text { clinical cultures }^{\mathrm{e}}\end{array}$ & $0.347(0.161$ to 0.532$)$ & $<0.001$ & $0.513(-0.206$ to 1.232$)$ & 0.162 & $-0.406(-0.594$ to -0.219$)$ & $<0.001$ \\
\hline Incidence density of MRSA ${ }^{f}$ & $0.025(-0.043$ to 0.092$)$ & 0.472 & $-0.137(-1.023$ to 0.750$)$ & 0.763 & $-0.067(-0.150$ to 0.011$)$ & 0.091 \\
\hline
\end{tabular}

INT, intervention; CI, confidence interval; CRAB, carbapenem-resistant Acinetobacter baumannii; ASC, active surveillance cultures; MRSA, methicillin resistant Staphylococcus aureus.

${ }^{a}$ Differences between first value after and last value before INT.

${ }^{\mathrm{b}}$ Monthly change after INT when compared with trend before INT.

${ }^{\mathrm{c}}$ Durbin-Watson statistic (DWS) $=1.847$.

${ }^{\mathrm{d}} \mathrm{DWS}=1.188$.

${ }^{\mathrm{e}} \mathrm{DWS}=1.203$.

${ }^{\mathrm{f}} \mathrm{DWS}=1.188$.

combined with CHG bathing resulted in successful control of CRAB infection or colonization in a medical ICU.

Restriction of carbapenem consumption is considered an important strategy to limit the spread of CRAB [4]. This recommendation is based on a series of studies that showed that previous carbapenem use was an important risk factor for multidrug-resistant (MDR) Acinetobacter infection, and its restriction with or without basic infection-control measures resulted in successful reduction of CRAB in both ICU and non-ICU settings [17-19]. Despite these recommendations, we chose not to impose any restrictions on antibiotic use in the ICU, which resulted in an increase in carbapenem use during period 2. It is often difficult to improve antibiotic use in ICUs because physicians are reluctant to withdraw or shorten antibiotic regimens in critically ill patients, and the complex decision-making process is sometimes made by doctors with limited expertise [20]. Given the lack of carbapenem restriction used in our ICU, this study clearly shows that a multifaceted intervention protocol is capable of reducing CRAB infection and colonization independent of antimicrobial use. However, despite the ultimate success of this approach, it still took nearly a year to bring the CRAB epidemic under control. This long delay may have been due to increased use of carbapenem during period 2, although definitive assessment of this potential association was beyond the scope of the present study.

We assumed that the combined interventions used in this study were complementary to one another for the following reasons. First, despite the increase in sampling sites for ASC in period 2, 21.8\% of CRAB-positive patients were not identified by surveillance cultures, consistent with results from a previous study showing the lack of sensitivity of CRAB-ASC [10]. Second, only a few studies have reported a positive association between CHG bathing and reduced CRAB acquisition rates, and all were single-center trials in different hospital settings, with different CHG bathing protocols and often evaluated as part of a larger infection-control effort [21,22]. Third, one post hoc analysis reported an additional benefit of universal glove and gown use for the reduction of MRSA acquisition when performing CHG bathing simultaneously, but this benefit has not been evaluated for CRAB [23]. As the multifaceted intervention used in period 2 included a feature of horizontal infection control strategies, the change in MRSA incidence was also evaluated, which showed a nonsignificant decrease in MRSA between the two periods. Therefore, the present study supports the implementation of horizontal infection-control measures including universal glove and gown use with CHG bathing when multiple MDR 
pathogens are epidemic or endemic in ICUs [7].

Through several meetings with the ICU nursing staff, our infection-control team continually emphasized the lack of sufficient evidence associated with any single advanced measure, thereby necessitating the use of a multifaceted control program, although this approach significantly increased nursing workloads. Universal glove and gown use was not initially included in the advanced programs, but was later introduced on the recommendation of the nursing staff. This change in institutional culture may have helped increase adherence to the basic control program (e.g., the significant increase in hand hygiene compliance in period 2) both in the short term, and for nearly 2 years thereafter, which were likely critical for the successful control of CRAB in our ICU [24].

There were several limitations to this study. First, we implemented a "bundle" of multiple recommended infection control measures, because no single intervention measure was likely to be successful in controlling CRAB infection or colonization in this epidemic [4]. Therefore, it was difficult to evaluate the relative contribution of each intervention program, because multiple interventions were implemented simultaneously or very close together. Second, although we analyzed the effects of multiple interventions on the control of CRAB acquisition by ITS using a segmented Poisson regression to estimate the association between the interventions and outcome, we could not control for the effects of potential confounders on the observed decrease in CRAB rates [15]. Third, because molecular typing was not performed, the epidemiological link of CRAB strains could not be determined. However, considering that most of the study patients were transferred from other hospitals or long-term care facilities, the coexistence of polyclonal outbreaks with endemic clones may explain the increase in the frequency of CRAB isolation in this study [25].

Taken together, the data presented here indicate that a multifaceted infection-control protocol including universal glove and gown use with daily CHG bathing can successfully reduce the incidence of CRAB infection or colonization in a medical ICU with no restrictions on carbapenem use. During our baseline control period, our standard infection-control program consisting of hand hygiene, ASCs, contact precautions, and environmental cleaning were not effective at controlling this difficult microorganism. The application of a more rig- orous multiple-intervention strategy should therefore be considered even in suggestive cases to prevent the establishment of endemic organisms.

\section{KEY MESSAGE}

1. For controlling a carbapenem-resistant Acinetobacter baumannii (CRAB) epidemic in a medical intensive care unit (MICU), we implemented an advanced intervention consisting of universal glove and gown use with daily chlorhexidine bathing in combination with basic infection control programs.

2. The multifaceted intervention led to a decrease in the incidence of CRAB from a peak of 17.65 to 0.89 cases per 1,000 patient-days in a MICU, where carbapenem was not restricted, during a 50-month study period.

\section{Conflict of interest}

No potential conflict of interest relevant to this article was reported.

\section{REFERENCES}

1. Munoz-Price LS, Weinstein RA. Acinetobacter infection. N Engl J Med 2008;358:1271-1281.

2. Apisarnthanarak A, Pinitchai U, Thongphubeth K, et al. A multifaceted intervention to reduce pandrug-resistant Acinetobacter baumannii colonization and infection in 3 intensive care units in a Thai tertiary care center: a 3-year study. Clin Infect Dis 2008;47:760-767.

3. Garnacho-Montero J, Dimopoulos G, Poulakou G, et al. Task force on management and prevention of Acinetobacter baumannii infections in the ICU. Intensive Care Med 2015;41:2057-2075.

4. Tacconelli E, Cataldo MA, Dancer SJ, et al. ESCMID guidelines for the management of the infection control measures to reduce transmission of multidrug-resistant Gram-negative bacteria in hospitalized patients. Clin Microbiol Infect 2014;20 Suppl 1:1-55.

5. Climo MW, Sepkowitz KA, Zuccotti G, et al. The effect of daily bathing with chlorhexidine on the acquisition of methicillin-resistant Staphylococcus aureus, vancomy- 
cin-resistant Enterococcus, and healthcare-associated bloodstream infections: results of a quasi-experimental multicenter trial. Crit Care Med 2009;37:1858-1865.

6. Harris AD, Pineles L, Belton B, et al. Universal glove and gown use and acquisition of antibiotic-resistant bacteria in the ICU: a randomized trial. JAMA 2013;310:1571-1580.

7. Huang SS, Septimus E, Kleinman K, et al. Targeted versus universal decolonization to prevent ICU infection. $\mathrm{N}$ Engl J Med 2013;368:2255-2265.

8. Heenen S, Jacobs F, Vincent JL. Antibiotic strategies in severe nosocomial sepsis: why do we not de-escalate more often? Crit Care Med 2012;40:1404-1409.

9. Su CH, Wang JT, Hsiung CA, et al. Increase of carbapenem-resistant Acinetobacter baumannii infection in acute care hospitals in Taiwan: association with hospital antimicrobial usage. PLoS One 2012;7:e37788.

10. Marchaim D, Navon-Venezia S, Schwartz D, et al. Surveillance cultures and duration of carriage of multidrug-resistant Acinetobacter baumannii. J Clin Microbiol 2007;45:1551-1555.

11. Ridenour G, Lampen R, Federspiel J, Kritchevsky S, Wong E, Climo M. Selective use of intranasal mupirocin and chlorhexidine bathing and the incidence of methicillin-resistant Staphylococcus aureus colonization and infection among intensive care unit patients. Infect Control Hosp Epidemiol 2007;28:1155-1161.

12. WHO Collaborating Centre for Drug Statistics Methodology. Guidelines for ATC Classification and DDD Assignment. Oslo: Norwegian Institute of Public Health, 2015 .

13. Clinical and Laboratory Standards Institute. Performance Standards for Antimicrobial Susceptibility Testing: Eighteenth Informational Supplement. Wayne: Clinical and Laboratory Standards Institute, 2008.

14. Wagner AK, Soumerai SB, Zhang F, Ross-Degnan D. Segmented regression analysis of interrupted time series studies in medication use research. J Clin Pharm Ther 2002;27:299-309.

15. Shardell M, Harris AD, El-Kamary SS, Furuno JP, Miller RR, Perencevich EN. Statistical analysis and application of quasi experiments to antimicrobial resistance intervention studies. Clin Infect Dis 2007;45:901-907.

16. Enoch DA, Summers C, Brown NM, et al. Investigation and management of an outbreak of multidrug-carbapenem-resistant Acinetobacter baumannii in Cambridge, UK. J Hosp Infect 2008;70:109-118.

17. Corbella X, Montero A, Pujol M, et al. Emergence and rapid spread of carbapenem resistance during a large and sustained hospital outbreak of multiresistant Acinetobacter baumannii. J Clin Microbiol 2000;38:4086-4095.

18. Lautenbach E, Synnestvedt M, Weiner MG, et al. Epidemiology and impact of imipenem resistance in Acinetobacter baumannii. Infect Control Hosp Epidemiol 2009;30:1186-1192.

19. Ogutlu A, Guclu E, Karabay O, Utku AC, Tuna N, Yahyaoglu M. Effects of Carbapenem consumption on the prevalence of Acinetobacter infection in intensive care unit patients. Ann Clin Microbiol Antimicrob 2014;13:7.

20. Luyt CE, Brechot N, Trouillet JL, Chastre J. Antibiotic stewardship in the intensive care unit. Crit Care 2014; 18:480.

21. Apisarnthanarak A, Pinitchai U, Warachan B, Warren DK, Khawcharoenporn T, Hayden MK. Effectiveness of infection prevention measures featuring advanced source control and environmental cleaning to limit transmission of extremely-drug resistant Acinetobacter baumannii in a Thai intensive care unit: an analysis before and after extensive flooding. Am J Infect Control 2014;42:116-121.

22. Chung YK, Kim JS, Lee SS, et al. Effect of daily chlorhexidine bathing on acquisition of carbapenem-resistant Acinetobacter baumannii (CRAB) in the medical intensive care unit with CRAB endemicity. Am J Infect Control 2015;43:1171-1177.

23. Morgan DJ, Pineles L, Shardell M, et al. Effect of chlorhexidine bathing and other infection control practices on the Benefits of Universal Glove and Gown (BUGG) trial: a subgroup analysis. Infect Control Hosp Epidemiol 2015;36:734-737.

24. Jain R, Kralovic SM, Evans ME, et al. Veterans affairs initiative to prevent methicillin-resistant Staphylococcus aureus infections. N Engl J Med 2011;364:1419-1430.

25. Marchaim D, Navon-Venezia S, Leavitt A, Chmelnitsky I, Schwaber MJ, Carmeli Y. Molecular and epidemiologic study of polyclonal outbreaks of multidrug-resistant Acinetobacter baumannii infection in an Israeli hospital. Infect Control Hosp Epidemiol 2007;28:945-950. 